\section{Severe episodic dyspnoea and abnormal flow-volume loop}

\section{CLINICAL PRESENTATION}

A 78-year-old never-smoking Caucasian woman presented with a 4-month history of persistent dry cough and episodic severe dyspnoea with inspiratory stridor and chest pain. She had visited the emergency department several times, where no significant abnormalities were noted. A suspected vocal cord dysfunction was excluded by fibreoptic laryngoscopy. Two months later spirometric examination showed moderate airway obstruction (forced expiratory volume in $1 \mathrm{~s} 64 \%$ of predicted) which was interpreted as asthma. After ineffective treatment with inhaled bronchodilator and corticosteroid, the patient was admitted to the hospital. Clinical examination, a chest

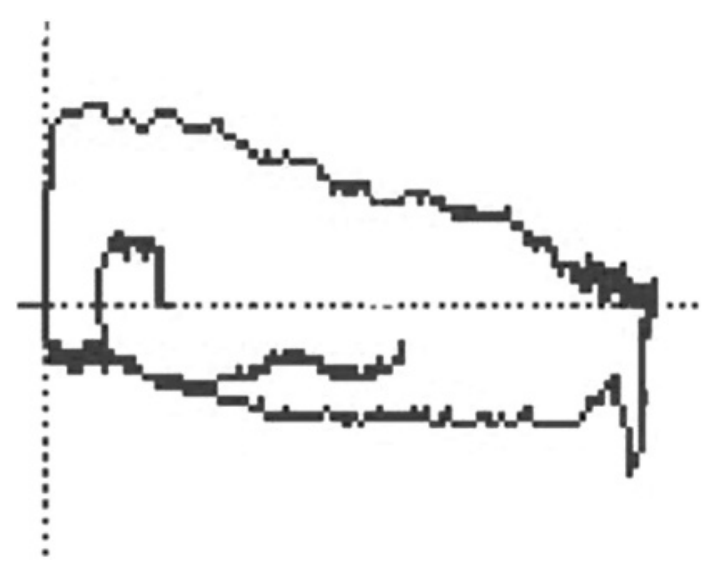

Figure 1 Flow-volume loop showing severe expiratory and inspiratory airflow limitation. radiograph and laboratory investigations were normal, apart from mild arterial hypoxaemia and mild hypogammaglobulinaemia. The ECG, transthoracic echocardiogram and cardiopulmonary exercise test were all normal.

Repeat spirometric examination confirmed a moderate airway obstruction which was unresponsive to bronchodilation. Analysis of the flow-volume loop (fig 1) showed fixed inspiratory and expiratory air flow limitation. Based on this finding, a chest CT scan (fig 2) was performed which showed a large solid mass $(6 \mathrm{~cm}$ wide and $8 \mathrm{~cm}$ length) enveloping the distal portion of the trachea, carina and the proximal tract of the main bronchi, with no involvement of lung parenchyma and no enlargement of the mediastinal and hilar lymph nodes.

Bronchoscopic examination demonstrated extrinsic compression of the major airways. Cytological and microbiological examinations of the lavage fluid were negative. Transbronchial biopsy showed monotonous proliferation of mature-appearing plasma cells, reactive lymphoid follicles and fibrosis (fig 3A) with multiple Russell bodies on PAS staining (fig 3B).

Immunoelectrophoresis revealed mild serum monoclonal IgG lambda component. Bone marrow biopsy and bone scintigram were normal.

\section{QUESTION}

What diagnosis do you suggest on the basis of the flow-volume loop, lung CT scan and biopsy findings?

See page 264 for answer.

This case was submitted by:

\section{Bucca, ${ }^{1}$ B Culla, ${ }^{1}$ S Asioli, ${ }^{1}$ F Rabbia, ${ }^{2}$ A Milan, ${ }^{2}$ G Limerutti, ${ }^{2}$ G Guida ${ }^{1}$}

${ }^{1}$ Department of Biomedical Sciences and Human Oncology, University of Turin, Italy; ${ }^{2}$ ASO S. Giovanni Battista, Turin, Italy

Correspondence to: Dr C Bucca, Department of Biomedical Sciences and Human Oncology, University of Turin, Italy; caterina.bucca@unito.it

Thorax 2009;64:210. doi:10.1136/thx.2008.100024
Figure 2 Chest CT scans showing a large solid mass in the middle mediastinum, enveloping the distal portion of the trachea, carina and the proximal tract of the main bronchi, causing significant narrowing of the airway lumen.
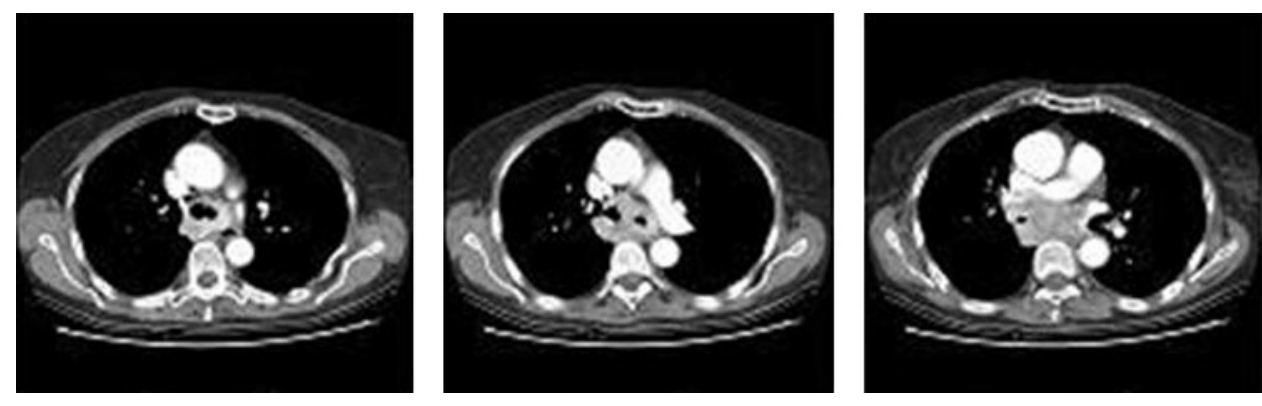

Figure 3 (A) H\&E-stained transbronchial specimen showing monotonous diffuse proliferation of small round cells without reactive lymph follicle or fibrosis. (B) PAS stain showing the presence of numerous Russell bodies.
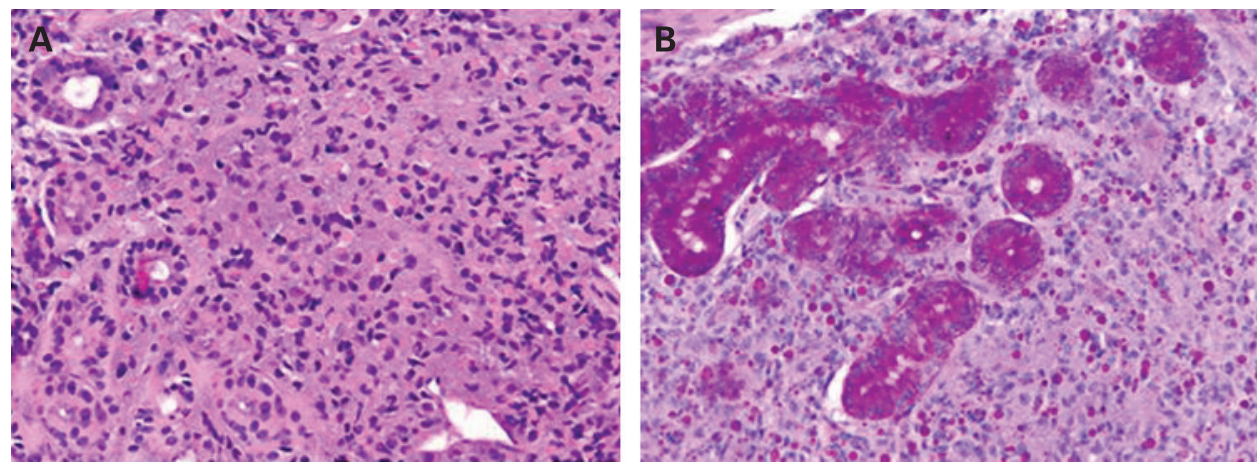